\title{
Selective leukemia cell death by activation of the double-stranded RNA-dependent protein kinase PKR
}

\author{
YA-JUAN LI ${ }^{1}{ }^{*}$, JIAN-MING ZENG $^{1}{ }^{*}$, SHI-FENG HUANG ${ }^{1}$, XIAO-ZHONG WANG $^{1}$, SHI-QIAO ZHAO ${ }^{1}$, \\ WEI-JUN BAI ${ }^{1}$, WEI-XI CAO ${ }^{1}$, ZONG-GAN HUANG ${ }^{2}$ and WEN-LI FENG ${ }^{1}$ \\ ${ }^{1}$ Department of Clinical Hematology, Key Laboratory of Laboratory Medical Diagnostics, Ministry of Education, \\ ${ }^{2}$ Department of Hematology, Clinic College, Chongqing Medical University, Chongqing 400016, P.R. China
}

Received December 21, 2010; Accepted March 2, 2011

DOI: $10.3892 / \mathrm{ijmm} .2011 .666$

\begin{abstract}
Deregulated activity of the BCR-ABL tyrosine kinase encoded by the Bcr-Abl oncogene represents an important therapeutic target for all the chronic myelogenous leukemia (CML) phases. In this study, we sought to identify targeted PKR activation by Bcr-Abl AS RNA, an anti-sense RNA complementary to the unique mRNA fragments flanking the fusion point of Bcr-Abl, which can be used as an effective anti-leukemia strategy in K562 cells. Moreover, we observed expression of Bcr-Abl AS RNA in K562 cells which resulted in selective apoptosis induction through specific activation of PKR, leading to phosphorylation of eIF2 $\alpha$, global inhibition of protein synthesis, caspase- 8 activation and BAX up-regulation. The targeted PKR activation and induced apoptosis were reversed by the PKR inhibitor 2-aminopurine. Taken together, our results indicate that targeted PKR activation led to selective apoptosis induction in K562 cells, which correlated with caspase- 8 activity and enhanced expression of BAX.
\end{abstract}

\section{Introduction}

The deregulated activity of the BCR-ABL tyrosine kinase (TK) encoded by the Bcr-Abl oncogene is a therapeutic target in all phases of chronic myelogenous leukemia (CML) (1-3). BCR-ABL activates numerous pro-growth and pro-survival mechanisms, which confer resistance of Bcr-Abl positive cells to apoptosis $(2,3)$. A comprehensive understanding of the molecular basis of CML has led to the development of highly effective and targeted therapies that inhibit BCR-ABL tyrosine kinase activity. The best example is represented by

Correspondence to: Professor Wen-Li Feng, Department of Clinical Hematology, Key Laboratory of Laboratory Medical Diagnostics, Ministry of Education, Chongqing Medical University, Chongqing 400016, P.R. China

E-mail: fengwlcqmu@sina.com

*Contributed equally

Key words: chronic myelogenous leukemia, dsRNA, PKR, K562 cells, BCR-ABL, apoptosis, caspase- 8 the treatment of CML with imatinib mesylate (STI571), a specifically designed inhibitor that targets the tyrosine kinase activity of the BCR-ABL and consequently induces apoptosis in CML cells and patients. However, patients may develop imatinib resistance, which may result from the amplification of Bcr-Abl, from mutations in the Abl kinase domain that lead to a drug-insensitive protein, or from Bcr-Abl-independent mechanisms (4-6). As a result, much interest is now focused on the development of other targeted therapeutic strategies in order to inflict a more effective blow upon CML cells at the time of blast crisis and resistance.

PKR, a double-stranded RNA-dependent protein kinase, first identified as a key molecule against viral infection, is now recognized not only as an important regulator of cell growth and cell differentiation, but also as an effective inducer of tumor apoptosis $(7,8)$. With strong serine/threonine kinase activity, active PKR in its phosphorylated form can activate the pro-apoptotic factors such as caspase- $8(9,10)$, caspase- 9 $(11,12)$, and Bax $(10)$, suggesting that PKR can be taken as an important target for tumor therapy. Therapeutic strategies aimed at targeting PKR are indeed beginning to offer promising results. Previous studies (13-15) have demonstrated that PKR can be activated in cancer cells by in situ generation of dsRNA in the cancer cell. The strategy is based on the fact that chromosomal translocations, the most common somatic mutation class among known oncogenes, lead to the production of unique mRNA species (16). Upon hybridization with the fusion mRNA by a designed antisense RNA of 30-85 nucleotide length, a dsRNA molecule will be generated that is long enough to activate PKR within cancer cells while the formed short dsRNA can not activate PKR in normal cells (17). PKR and its signaling pathway are not restricted to a given cell line; therefore, in principle, this dsRNA-mediated sacrificing strategy (DKS) can be applied to any cancer that expresses unique RNA sequences. As a result, we believe that a treatment strategy aiming at sequence-specific activation of PKR in Bcr-Abl positive cells is of promising potential in treating CML with minor or no toxic effects on normal cells.

\section{Materials and methods}

Chemicals. M-MLV reverse transcriptase and TaqDNA polymerase were purchased from Promega. TaqPlus, the restriction 
Table I. Sequences synthesized to construct the retroviral vectors.

\begin{tabular}{ll}
\hline Name & \multicolumn{1}{c}{ Sequence } \\
\hline Bcr-Abl AS & 5'-GATCCACTGGCCGCTGAAGGGCTTcTGAACTCTGCTTAAATCCTTTTTTGGAAA-3' (54 nt) \\
Bcr-Abl S & 5'-AGCTTTTCCAAAAAAGGATTTAAGCAGAGTTCAGAAGCCCTTCAGCGGCCAGTG-3' (54 nt) \\
EcoRI-GFP & 5'-CTAGAATTCAACCATGGTGAGC-3' (22 nt) \\
Xhol-GFP & 5'-GCACTCGAGCTTTACTTGTAC-3' (21 nt) \\
Sall-H1 & 5'-GTCGTCGACGGTACCGAATTCAT-3' (23 nt) \\
HindIII-H1 & 5'-CCGCTCGAGAAGCTTTTCC-3' (19 nt)
\end{tabular}

AS, antisense; S, sense. Bold type indicates the thymidine residue at position 25 of Bcr-Abl AS which was replaced by cytosine.

Table II. Sequences of PCR primers and the anticipated base pair sizes of the PCR products.

\begin{tabular}{llc}
\hline Primers & \multicolumn{1}{c}{ Sequence } & PCR product size \\
\hline Bcl-2 forward & 5'-CGACTTCGCCGAGATGTCCAGCCAG-3' & $388 \mathrm{bp}$ \\
Bcl-2 reverse & 5'-ACTTGTGGCCCAGATAGGCACCCAG-3' \\
Bax forward & 5'-GATGCGTCCACCAAGAAGCT-3' & $170 \mathrm{bp}$ \\
Bax reverse & 5'-CGGCCCCAGTTGAAGTTG-3' & \\
$\beta$-actin forward & 5'-GTGGACATCCGCAAAGAC-3' & $302 \mathrm{bp}$ \\
$\beta$-actin reverse & 5'-AAAGGGTGTAACGCAACTAA-3' &
\end{tabular}

enzymes EcoRI, XhoI, SacI, HindIII and the DNA ligation kit were purchased from Takara. OligodT and dNTPs were from Shanghai Sangon company. RPMI-1640 culture media was from Hyclone. Fetal bovine serum was purchased from Hangzhou Sijiqing Biological Engineering Material Co., Ltd. Glutamine, the PKR inhibitor, 2-aminopurine (2-AP) and polybrene were obtained from Sigma. L-liposome DMRIE-C, synthesized dsRNA analogs and polyIC were from Invitrogen. The caspase- 8 inhibitor, Z-IETD-FMK, was produced by Biovision. The pre-stained protein marker, acrylamide, N,N'methylene bisacrylamide, polyvinylidene fluoride (PVDF) membranes, bromophenol blue, and glycocine were purchased from Beijing Biotech Co., Ltd. The pMSCV-neo retroviral vector was purchased from BD Biosciences. The pSilencer3.0H1 was from Ambion. The IRES2-EGFP was kindly provided by Dr Zuo Guowei in the Ultrasound Engineering Research Institute of Chongqing Medical University.

Construction of Bcr-Abl AS RNA-encoding retroviral vector. The $38 \mathrm{nt}$ antisense (AS) and sense (S) oligonucleotides corresponding to both $19 \mathrm{nt}$ fragments of Bcr-Abl immediately flanking the fusion region were synthesized, self-annealed and inserted into the Bam HI/HindIII sites of the pSilencer3.0H1. Upon hybridization with wild-type Bcr and Abl, the AS RNA produces two dsRNA fragments each of $19 \mathrm{bp}$, while with Bcr-Abl it produces a 38 bp dsRNA. The transcription termination signal for RNA polymerase III is a string of four or more thymidine residues (19). To prevent early termination of transcription, the thymidine residue at position 25 of Bcr-Abl AS was replaced by cytosine (indicated by the lowercase bold type letter in Table I). As binding and activation of PKR by dsRNA can tolerate a single-base mismatch (18), the above replacement should not interfere with PKR activation. The pMSCV-GFP vector was derived from pMSCV-neo by replacement of neo with a GFP cassette, which was amplified from pIRES2-EGFP. For retroviral vector construction, the $\mathrm{H} 1$ cassette was amplified and subcloned into the SalI/HindIII sites of pMSCV-GFP to obtain pMSCV/GFP-H1-BCR/ ABL 38AS vector. The selected clone was confirmed by DNA sequencing analysis. All the sequences synthesized to construct the retroviral vectors are shown in Table I.

Cell culture and retrovirus transduction. K562 cells and normal control ECV304 cells were obtained from the American Type Culture Collection (Manassas, VA, USA) and maintained in RPMI-1640 medium and DMEM medium respectively, supplemented with $10 \%$ fetal calf serum, $2 \mathrm{mM}$ glutamine and antibiotics (100 U/ml penicillin and $100 \mu \mathrm{g} / \mathrm{ml}$ streptomycin) at $37^{\circ} \mathrm{C}$ in a humidified atmosphere of $5 \% \mathrm{CO}_{2}$. Liposome DMRIE-C was used to transfect polyIC into K562 and ECV304. Retrovirus containing pMSCV-GFP and pMSCV/ GFP-H1-Bcr-Abl 38AS were generated by transfection of these constructs into PT67 cells using liposome DMRIE-C. Virus supernatants were harvested at $48 \mathrm{~h}$, passed through a $0.45 \mu \mathrm{m}$ filter, aliquoted and kept at $-80^{\circ} \mathrm{C}$. The generated virus titer was $5.5-7.0 \times 10^{5} \mathrm{cfu} / \mathrm{ml}$ and the stocks were designated as RV-GFP and RV-38AS respectively. The retroviral transduction was done per the recommended protocols provided by BD Biosciences.

Morphological analysis. K562 cells were infected with RV-GFP and RV-38AS for $24 \mathrm{~h}$, collected, cytospined, stained with Wright-Giemsa and observed for nuclear and morphological changes under a microscope. 
Cell cycle analysis. Cells were harvested, washed with cold PBS, fixed in $70 \%$ ethanol and stored at $4{ }^{\circ} \mathrm{C}$. Cells were treated with RNase-A solution $(500 \mathrm{U} / \mathrm{ml})$ at $37^{\circ} \mathrm{C}$ for $15 \mathrm{~min}$ and stained by propidium iodide $(50 \mu \mathrm{g} / \mathrm{ml})$ in $1.12 \%$ sodium citrate at room temperature before analysis. Flow cytometric determination of DNA content was analyzed by the Coulter Epics XL Flow Cytometer (Coulter Corporation, Miami, FL, USA). The fractions of cells in the $G_{0} / G_{1}, S$ and $G_{2} / M$ phase were assessed using the cell cycle analysis software, Multicycle (Phoenix Flow System, San Diego, CA, USA).

Apoptosis analysis. Cells were collected, washed with cold PBS, fixed with ethanol and stained with PE-labeled Annexin-V (Responsif, Erlangen, Germany) and 7-AAD. Apoptosis was determined by the FACSCalibur flow cytometer (BD Biosciences, San Jose, CA, USA) and data analysis was performed with the CellQuest software (BD Biosciences). DNA laddering was detected by isolating fragmented DNA using the SDS/proteinase K/RNase A extraction method, which allows the isolation of fragmented DNA without the contamination from genomic DNA (20).

Transmission electron microscopy. Cells were collected and fixed in $2.5 \%$ glutaraldehyde solution. Fixed cells were washed three times with cold cacodylate solution, and postfixed with $0.1 \mathrm{~mol} / 1$ cacodylate buffer for $3 \mathrm{~h}$ at $4^{\circ} \mathrm{C}$. After washing with distilled water, cells were stained en bloc with uranyl acetate, dehydrated in graded ethanol and propylene oxide, infiltrated, embedded, and polymerized in polybed resin. Cells were then infiltrated for $6 \mathrm{~h}$ with $100 \%$ polybed resin, embedded in fresh resin, and polymerized overnight at $70^{\circ} \mathrm{C}$. Thin sections were cut, stained with uranyl acetate and lead citrate, viewed and photographed with a Hitachi-600 transmission electron microscope.

Assays of caspase-8 and caspase-9 activities. The detection of the activities of caspase- 8 and caspase- 9 were carried out per the manufacturer's instructions for the quantitative caspase- 8 and caspase-9 activity detection kit (Nanjing KeyGen Biotech Co., Ltd., China).

$R N A$ preparation and RT-PCR analysis. The total RNA was extracted by TRIzol reagents (Takara) according to the manufacturer's instructions and approximately $20 \mu \mathrm{g}$ total RNA were subjected to electrophoresis on a $1 \%$ agarose gel to verify the quality of the RNA. RT-PCR assays for Bax and $\mathrm{Bcl}-2$ were performed using standard conditions and the product was normalized to the control gene, $\beta$-actin. The sequences of the PCR primers are listed in Table II.

Protein translation detection. Cells were seeded into a 24-well plate at a density of $5 \times 10^{5} / \mathrm{ml}$ and grown overnight. Cells were then infected with 400,000 IU of appropriate retrovirus and treated with $5 \mathrm{mM}$ of 2 -AP where indicated. After $24 \mathrm{~h}$, cells were washed twice with PBS and serum-free medium was added. After $6 \mathrm{~h}, 1 \mu \mathrm{Ci}{ }^{3} \mathrm{H}$-leucine was added for $20 \mathrm{~h}$, and cells were then washed twice with PBS and collected. Equal volumes were taken for determination of total protein labeling and total mRNA concentration. Cells were lysed using $100 \mu \mathrm{l}$ of cell lysis buffer. Lysates were then
Table III. Effects of Bcr-Abl AS and the PKR inhibitor, 2-AP, on the cell cycle distribution of the K562 cell population.

\begin{tabular}{lccc}
\hline Group & $\mathrm{G}_{0} / \mathrm{G}_{1}(\%)$ & $\mathrm{S}(\%)$ & $\mathrm{G}_{2} / \mathrm{M}(\%)$ \\
\hline UT & 36.44 & 58.53 & 5.03 \\
AS & $57.47^{\mathrm{a}}$ & $31.48^{\mathrm{a}}$ & $11.05^{\mathrm{a}}$ \\
IC & $50.97^{\mathrm{a}}$ & $37.26^{\mathrm{a}}$ & $11.76^{\mathrm{a}}$ \\
RG & 36.22 & 58.07 & 5.71 \\
AS+2-AP & 37.88 & 56.02 & 6.10 \\
\hline
\end{tabular}

K562 cells were infected with Bcr-Abl AS with or without pre-treatment with the PKR inhibitor, 2-aminopurine (2-AP), or transfection with polyIC. The cells were harvested, stained with propidium iodide and analyzed by flow cytometry. Data shown are the means of three duplicate experiments. UT, untreated group; AS, RV-38AS-treated group; IC, polyIC-treated group; RG, RV-GFP-treated group; $\mathrm{AS}+2-\mathrm{AP}, \mathrm{RV}-38 \mathrm{AS}+2$-AP-treated group. ${ }^{\mathrm{a}} \mathrm{P}<0.05$ as compared with the untreated group.

transferred to scintillation vials and the radioactivity was counted in an LS6500 $\beta$-counter.

Western blot analysis. Approximately $1 \times 10^{6}$ cells were pelleted and lysed with $200 \mu \mathrm{l}$ modified RIPA buffer $(50 \mathrm{mM}$ Tris- $\mathrm{HCl}$, $150 \mathrm{mM} \mathrm{NaCl}, 1 \%$ Igepal CA-630, 0.25\% Na-desoxycholate, $1 \mathrm{mM}$ EDTA) supplemented with a protease inhibitor cocktail for $60 \mathrm{~min}$ on ice. The lysates were centrifuged at $14,000 \mathrm{x} \mathrm{g}$ for $30 \mathrm{~min}$ at $4^{\circ} \mathrm{C}$, after which the supernatants were collected and stored at $-80^{\circ} \mathrm{C}$. Protein concentration was determined using the BCA protein assay. For each sample, the same amount of proteins were denatured and applied onto SDS-PAGE gels; separated proteins were transferred to PVDF membranes followed by blocking with $10 \%$ non-fat dry milk. The membranes were incubated with primary antibodies against PKR, p-PKR, BCL-2 and BAX (Santa Cruz Biotechnology), followed by incubation with an HRP-conjugated secondary antibody (Wuhan Boshide Company, China). The blots were developed by DAB (Beijing Biotech Co., Ltd.), imaged and analyzed by photodensitometry using the Bio-Rad Gel Imaging System. The membranes were re-probed with an antibody against $\beta$-actin (Santa Cruz Biotechnology) using the same procedures described above.

Statistical analysis. Data are expressed as means \pm standard error of the mean (SEM). Statistical analyses were performed by unpaired Student's t-tests. A P-value of $\leq 0.05$ was taken to indicate statistical significance.

\section{Results}

Expression of Bcr-Abl AS RNA induces cell cycle arrest and apoptosis in K562 cells. The effects of Bcr-Abl AS RNA were assessed in vitro, and we observed that expression of Bcr-Abl AS RNA or transfection with polyIC led to cell cycle arrest in K562 cells (Table III). For example, RV-38AS treatment for $24 \mathrm{~h}$ increased the percentage of cells in the $\mathrm{G}_{0} / \mathrm{G}_{1}$ phase from 36.44 to $57.47 \%$. Concomitant with this increase was a significant decrease in the percentage of cells 
A

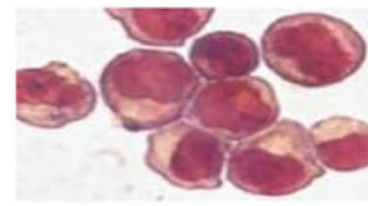

UT $(\times 600)$

B

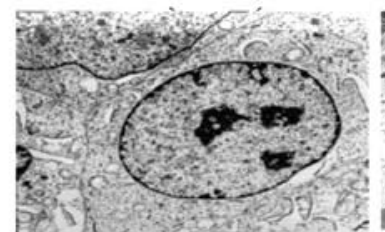

UT $(\times 6000)$

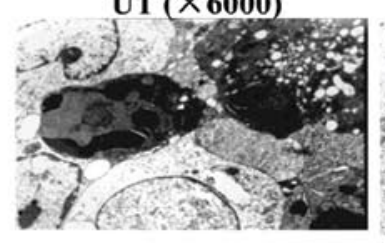

AS $(\times 6000)$

C

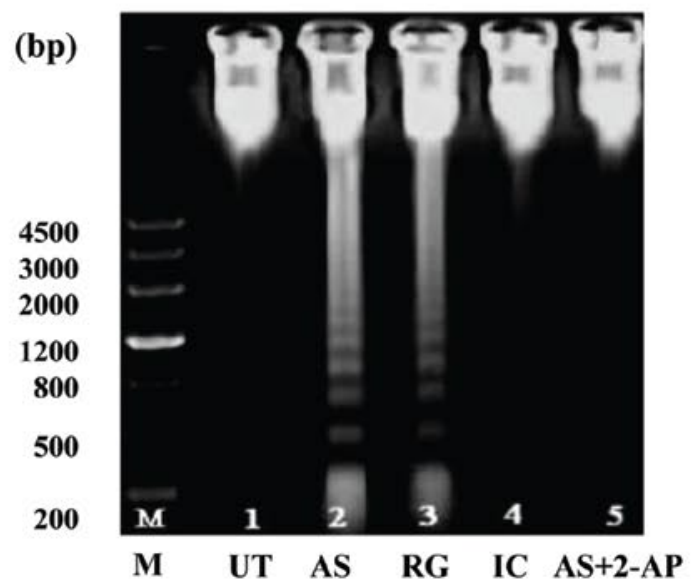

D

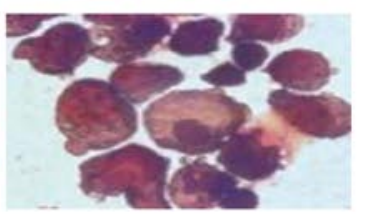

AS $(\times 600)$

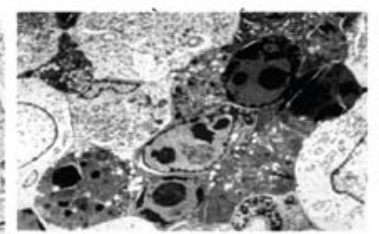

AS $(\times 6000)$

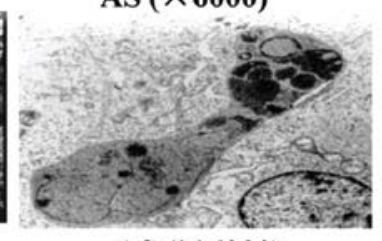

AS $(\times 6000)$
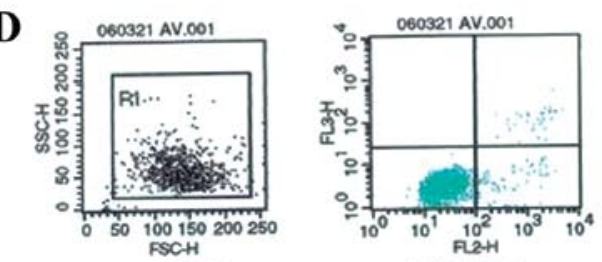

A
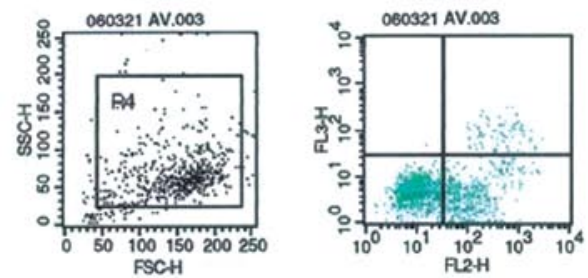

Flle: 060321 AV.001


C

File: 060321 AV.002

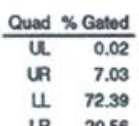

\begin{tabular}{ll}
12.39 \\
\hline$R$
\end{tabular}
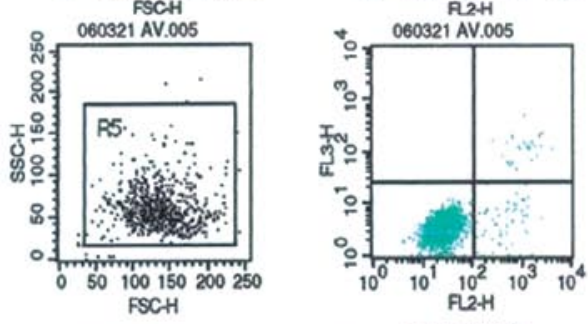

File: 060321 AV.003
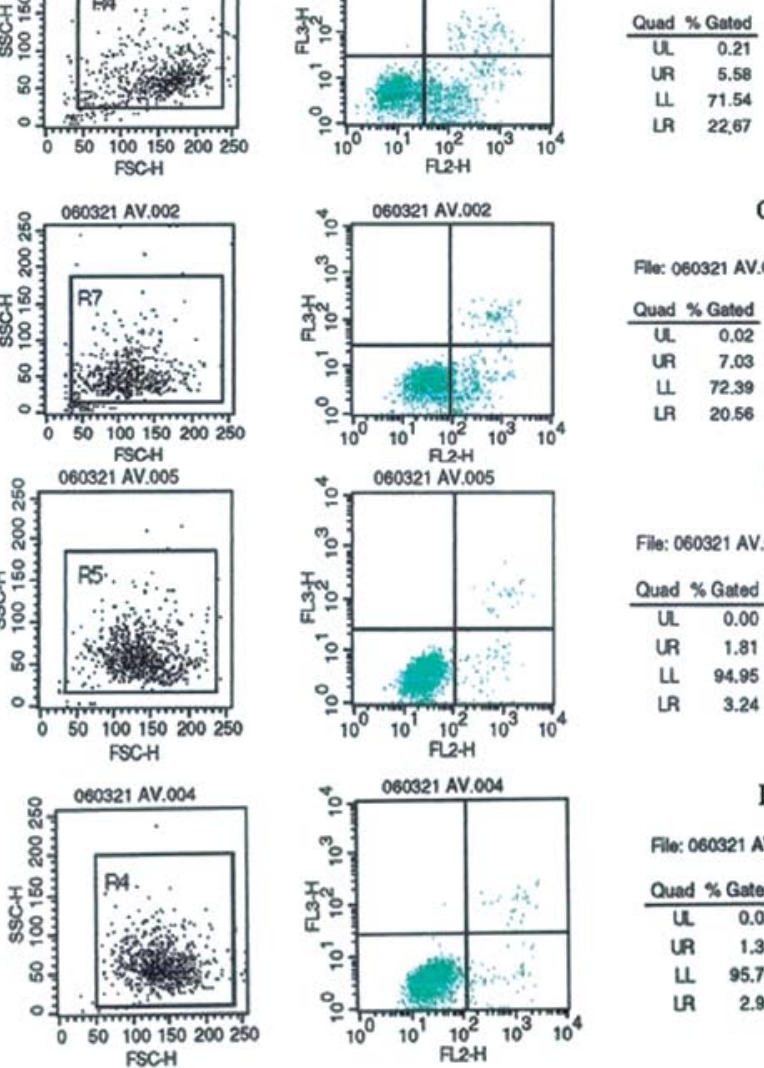

E

File: 060321 AV.004

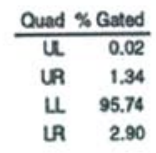

Figure 1. RV-38AS-induced apoptosis in K562 cells. Apoptosis of K562 cells observed by (A) light microscopy and (B) transmission electronic microscopy. (C) Detection of the fragmented DNA. Cells either untreated (lane 1), transfected with polyIC (lane 3), infected with the appropriate retrovirus (lane 2 and 4) or treated with 2-AP (lane 5). (D) Flow cytometry analysis. UT, untreated group; AS, RV-38AS-treated group; IC, polyIC-treated group; RG, RV-GFP-treated group; AS+2-AP, RV-38AS + 2-AP-treated group.

in the S phase from 58.53 to $31.48 \%$. Furthermore, the addition of 2-AP reversed this cell cycle arrest (Table III). As is shown in Fig. 1, expression of Bcr-Abl AS RNA induced nuclear condensation, cell shrinkage and membrane blebbing as could readily be observed under a light microscope (Fig. 1A). Apoptotic bodies were observed under a transmission electron microscope (Fig. 1B). Moreover, it was found that RV-38AS induced an accumulation of fragmented DNA (Fig. 1C), a hallmark of apoptotic cell death. Flow cytometric analysis further confirmed the massive apoptosis induced by the expression of Bcr-Abl AS RNA (Figs. 1D and 2A). We further demonstrated that expression of Bcr-Abl AS RNA induced apoptosis in K562 cells in a time-dependent manner (Fig. 2B). These results suggest that after cell cycle arrest, RV-38AStreated cells may undergo apoptosis. This possibility was supported by the findings in which the addition of RV-38AS to K562 cells resulted in elevated activities of both caspase- 8 and caspase-9 with time kinetics similar to the RV-38AS-induced loss of cell viability (Fig. 4A).
Expression of Bcr-Abl AS RNA activates PKR in Bcr-Abl positive K562 cells, but not in Bcr-Abl negative cells. In order to confirm specific PKR activation in Bcr-Abl positive K562 cells, we first examined PKR activity in both K562 and ECV304 cells. As shown in Fig. 3A, infection of K562 cells with RV-38AS led to strong PKR activation that was comparable to that of cells transfected with the synthetic dsRNA molecule polyIC, a universal activator of PKR. In contrast, PKR activity in ECV304 cells infected with RV-38AS exhibited no detectable changes compared to that of untreated cells. The total protein expression levels of PKR in both cells were not altered by the expression of Bcr-Abl AS RNA, indicating that the activation of PKR induced by Bcr-Abl AS RNA expression is specific to Bcr-Abl positive cells.

Bcr-Abl AS RNA-induced apoptosis is mediated through the PKR activation signaling pathway in K562 cells. To study the involvement of PKR and to further clarify the role of PKR activation in Bcr-Abl AS RNA-induced apoptosis in K562 


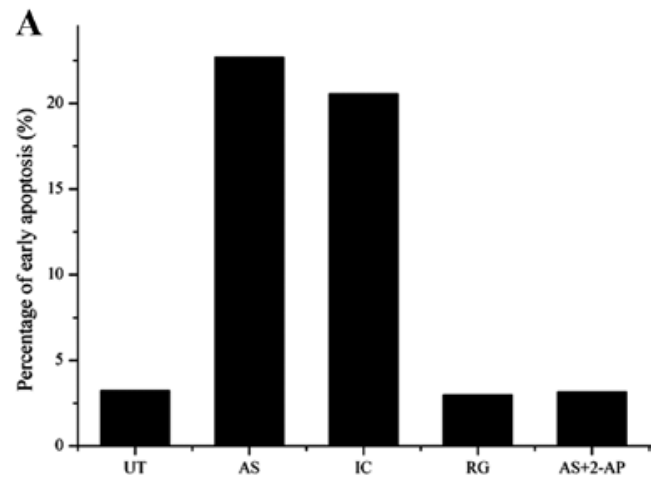

B

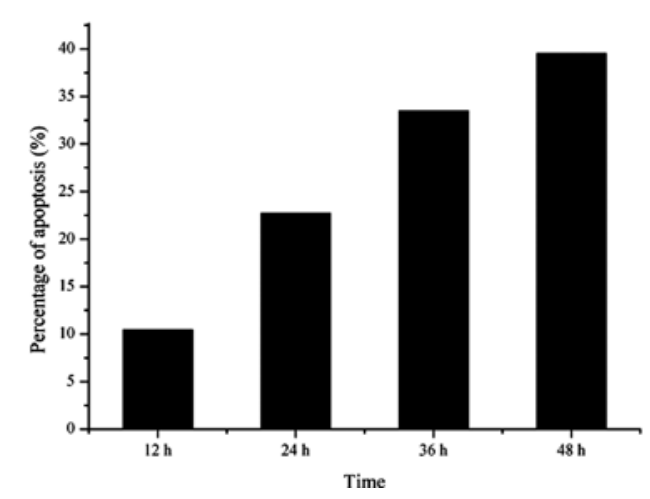

Figure 2. Expression of Bcr-Abl-AS RNA induces time-dependent apoptosis in K562 cells assessed by flow cytometry. (A) Apoptosis of K562 cells under different treatments. (B) RV-38AS induces K562 cell death in a time-dependent manner. UT, untreated group; AS, RV-38AS-treated group; IC, polyIC-treated group; RG, RV-GFP-treated group; AS+2-AP, RV-38AS + 2-AP-treated group.

cells, we examined the effect of the specific PKR inhibitor, 2-AP, on PKR activation and apoptosis induction. Treatment with 2-AP reversed the RV-38AS-induced PKR activation (Fig. 3A). Meanwhile, as shown in Fig. 1C and D and Fig. 2A, the results from both DNA gel electrophoresis and flow cytometric analysis of cell apoptosis revealed that RV-38ASinduced apoptosis was effectively inhibited by 2 -AP.

It has been shown that the events immediately downstream of PKR activation involved in apoptosis are the phosphorylation of eIF $2 \alpha$ by PKR and the activation of NF- $\mathrm{BB}$ transcription factors $(21,22)$. Our results showed that infection with RV-38AS also stimulated phosphorylation of endogenous eIF2 $\alpha$ in K562 cells, but not in ECV304 cells (Fig. 3A), further confirming the specificity of the PKR activation in Bcr-Abl positive cells.

We next examined whether PKR activation-induced eIF $2 \alpha$ phosphorylation leads to a global inhibition of protein synthesis. Infection of K562 cells with RV-38AS resulted in a significant reduction in total protein translation (Fig. 3B). The reduced protein translation was reversed through treatment of the RV-38AS-infected cells with 2-AP, suggesting that the PKR/eIF2 $\alpha$ pathway may be directly involved in the inhibition of the translation. These results confirm that RV-38AS-induced apoptosis is mediated through the PKR activation signaling pathway in K562 cells.

Effects of PKR activation on caspase activation. Earlier studies have shown that PKR activation induces apoptosis of
A

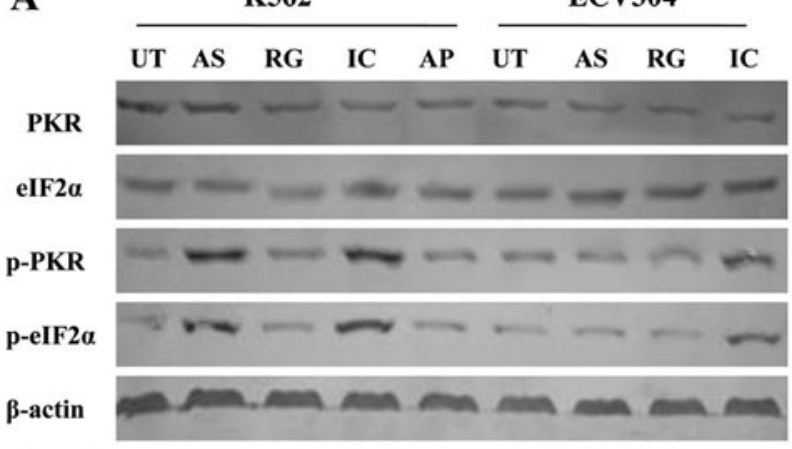

B

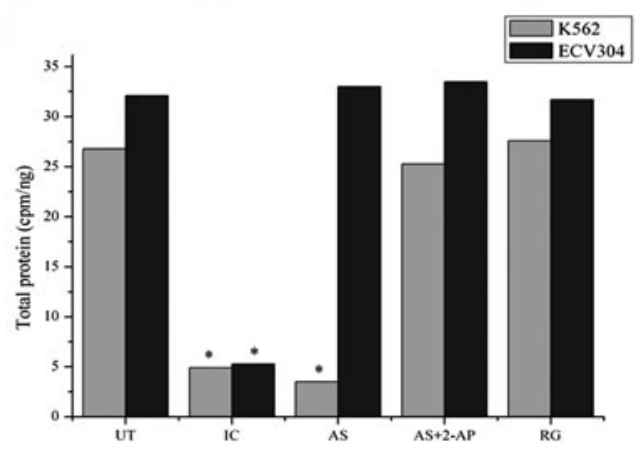

Figure 3. Involvement of PKR in the apoptosis of K562 cells. (A) Activation of PKR in K562 and ECV304 cells. (B) Effect of RV-38AS infection on the total protein translation in K562 and ECV304 cells. ${ }^{*} \mathrm{P}<0.05$ as compared with untreated group. UT, untreated group; AS, RV-38AS-treated group; IC, polyIC-treated group; RG, RV-GFP-treated group; AS+2-AP, $\mathrm{RV}-38 \mathrm{AS}+2$-AP-treated group.

mammalian cells through activation of the caspase pathways (9-12). We sought to examine the possible involvement of caspase- 8 and caspase- 9 during the apoptosis process induced by the expression of Bcr-Abl AS RNA. ELISA assays showed that activation of PKR in K562 cells resulted in the elevated activation of both caspase- 8 and caspase- 9 as early as $4 \mathrm{~h}$, and their peak values were respectively reached at 12 and $18 \mathrm{~h}$ (Fig. 4A). In the presence of the caspase- 8 specific inhibitor, Z-IETD-FMK, RV-38AS-induced caspase-8 activity was significantly reduced at $4 \mathrm{~h}$ (Fig. 4A), whereas the activity of caspase- 9 was only partially reduced, indicating that the RV-38AS induced apoptosis may be mediated, at least in part, through a caspase- 8 dependent pathway. As caspase- 8 is up-stream of caspase- 9 activation, this is in good concordance with the findings that downstream caspase-9 is activated by caspase- 8 during PKR-induced cell apoptosis $(11,12)$. Conversely, we found that the activation of both caspase- 8 and caspase- 9 was attenuated after treatment with 2-AP in K562 cells (Fig. 4A), indicating the PKR activation-dependent effect of Bcr-Abl AS RNA on caspase activation. Consistent with its inhibitory effect on PKR activation (Fig. 3A left) and the activities of caspase- 8 and caspase-9 (Fig. 4A), 2-AP was shown to prevent RV-38AS-induced apoptosis (Fig. 1C and D). The data further support that PKR activation plays an important role in apoptosis induction and caspase activation in K562 cells infected with RV-38AS. Thus, our findings strongly suggest that RV-38AS-induced apoptosis in K562 cells may be mediated, at least in part, by the PKR/ caspase- 8 signaling pathway. 
A
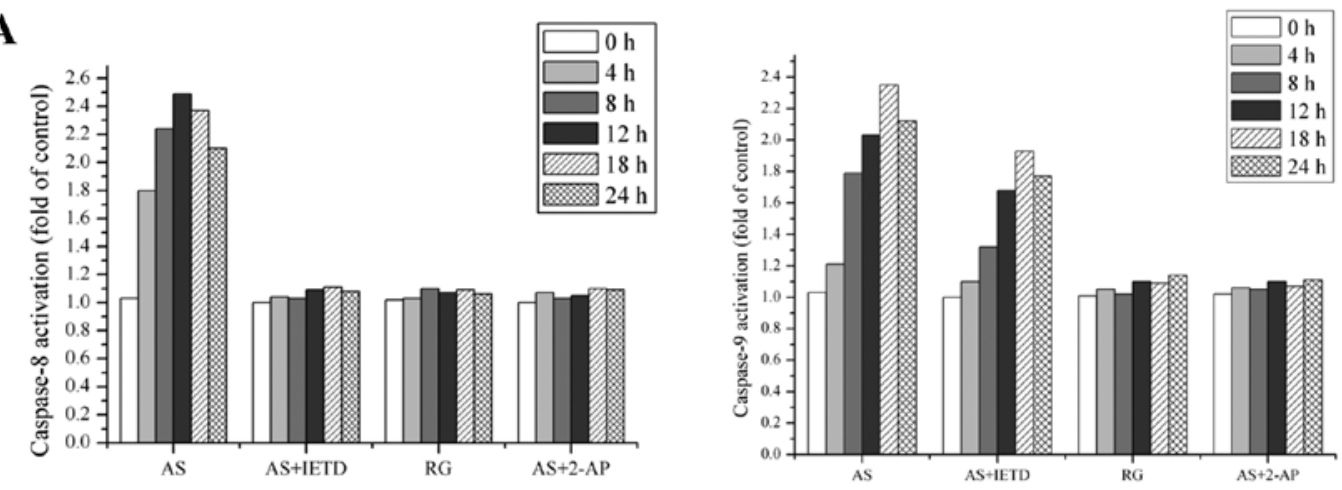

B

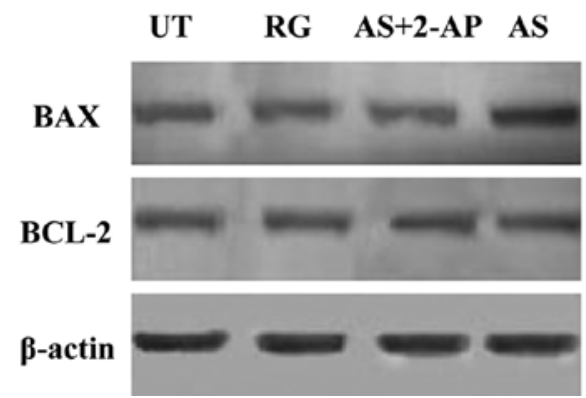

C

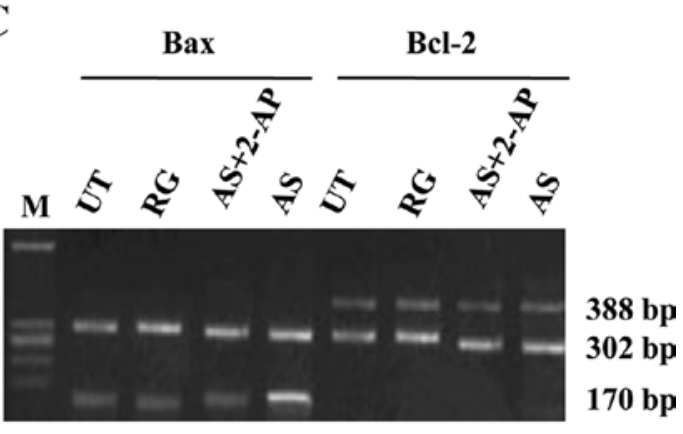

Figure 4. Involvement of caspases and Bax in apoptosis of K562 cells induced by Bcr-Abl AS RNA. (A) Effect of Bcr-Abl AS RNA on caspase-8 and caspase-9 activation. Cells were treated with appropriate retroviruses with or without the caspase-8 inhibitor, IETD, or the PKR inhibitor, 2-AP, for different time periods as indicated. Data shown are the means of three duplicate experiments. (B) Western blot analyses of the protein expression levels of BAX and BCL-2 in K562 cells. (C) RT-PCR analysis of mRNA expression levels of Bax and Bcl-2 in K562 cells. Cells were treated as indicated. The amount of $\beta$-actin was used as an internal control. Data shown are representative of three independent experiments with similar results. Marker: X-174/HaeIII DNA ladder. UT, untreated group; AS, RV-38AS-treated group; IC, polyIC-treated group; RG, RV-GFP-treated group; AS+2-AP, RV-38AS + 2-AP-treated group.

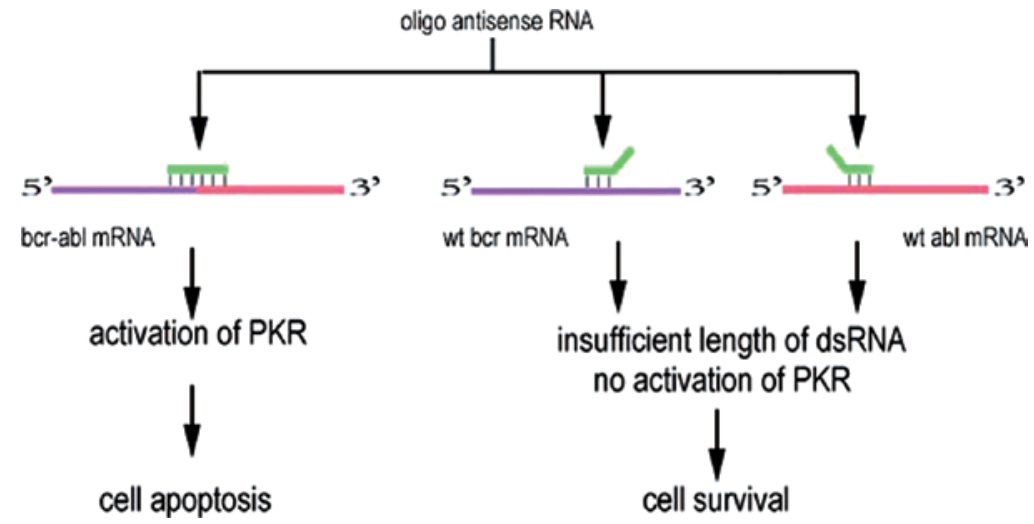

Figure 5. Mechanism of sequence-specific activation of PKR in K562 cells. Oligo anti-sense RNA of 38 nt, complementary to fragments flanking the fusion site in Bcr-Abl, can produce a dsRNA molecular of $38 \mathrm{bp}$, a sufficient length to activate PKR and induce cell apoptosis upon hybridization with Bcr-Abl, but not wild-type Bcr or Abl.

Effects of PKR activation on the protein expression of BCL-2 and BAX. The BCL-2 superfamily members play an important role in the regulation of the structural and functional stability of the mitochondrial membrane, and are known to be a 'check point' in the apoptosis process at mitochondrial level. Apoptosis induced by caspase activation is regulated by the balance between BCL-2 and BAX. To verify whether the expression of BCL-2 and BAX was involved in PKR activation-induced K562 cell apoptosis, we conducted RT-PCR and Western blotting experiments to evaluate their expression. Our results illustrated that PKR activation up-regulated BAX both at the mRNA and protein level, while the expression of
BCL-2 was not affected (Fig. 4B and C). These results indicate that a significant decrease in the BCL-2/BAX ratio, due to up-regulation of BAX and the lack of change in the BCL-2 expression, was observed in K562 cells treated with RV-38AS compared to untreated cells. The Bcr-Abl AS RNA-induced up-regulation of $\mathrm{BAX}$ at both the protein and mRNA level were reversed by the addition of 2-AP (Fig. 4B and C).

\section{Discussion}

In this study, we have demonstrated that the selective cell cycle arrest and apoptosis induction of K562 cells can be 
achieved through a specific stimulation of PKR activity by the expression of a unique dsRNA, a 38-nucleotide long antisense RNA complementary to the unique e14a2 junction of Bcr-Abl in K562 cells. As is shown in Fig. 5, the oligo anti-sense RNA of $38 \mathrm{nt}$, complementary to fragments flanking the fusion site in Bcr-Abl, can produce a dsRNA molecule of $38 \mathrm{bp}$, a sufficient length to activate PKR and induce cell apoptosis upon hybridization with Bcr-Abl fusion mRNA, but not wild-type Bcr or Abl, leading to sequence-specific activation of PKR in Bcr-Abl positive cells. We further found that expression of 38AS-RNA caused cell cycle arrest and massive apoptotic cell death in K562 cells, but not in ECV304 cells which express the wild-type Bcr and Abl. PKR was shown to be specifically activated in K562 cells with elevated caspase- 8 and caspase-9 activity and an enhanced expression of BAX, which was shown to be inhibited by 2 -AP. Taken together, our data suggest that the expression of Bcr-Abl AS RNA specifically and effectively induces apoptosis in K562 cells, which correlates well with the targeted activation of PKR, the subsequent activation of caspases and the up-regulation of BAX. Our results further indicate that the dsRNA-mediated cell killing strategy may be useful in treating any cancers that express a unique RNA species.

Although in principle, the dsRNA-mediated cell killing strategy may be useful in treating any cancers types that express a unique RNA species, so far, PKR activation-induced apoptosis by this mechanism had successfully been used only in some solid tumor cells $(13,14,23)$. In the present study, we investigated the feasibility of the specific activation of PKR and the selective induction of apoptosis in K562 cells through the expression of Bcr-Abl AS RNA, and further explored the underlying mechanisms leading to apoptosis. Elevated phosphorylation of both PKR and its well-known key substrate, eIF2 $\alpha$, in K562 cells but not in ECV304 cells, indicated the selective activation of PKR in K562 cells. To determine whether Bcr-Abl AS RNA induces apoptosis through PKR activation, an antagonist of PKR, 2-AP, was employed. Co-treatment with 2-AP rescued K562 cells from apoptosis induced by the expression of Bcr-Abl AS RNA. The findings that Bcr-Abl AS RNA selectively activated PKR and that 2-AP abrogated apoptosis induced by Bcr-Abl AS RNA indicate that expression of Bcr-Abl AS RNA could specifically induce apoptosis in K562 cells through selective activation of PKR in Bcr-Abl positive cells.

Earlier studies $(24,25)$ have demonstrated that PKR activity varies during cell cycle progression, with highest levels in early $G_{1}$ and a second peak at the $G_{1} / S$ boundary. Cells expressing the catalytic inactive form of PKR or lacking PKR have a prolonged $G_{1}$ phase, with fewer cells engaged in the $S$ phase, suggesting that PKR may have a role in cell cycle progression. Concurring with these observations, our results showed that after infection of K562 cells with RV-38AS, the percentage of cells in the $\mathrm{S}$ phase drops to about half of the number found in the untreated control group, which was further shown to be reversed by 2-AP, indicating that PKR activation induced $\mathrm{G}_{1}$ cell cycle arrest in K562 cells. Cell cycle arrest triggers programmed cell death in many tumor cell lines. As shown in Fig. 4A, this is also applicable to PKR activation in K562 cells. Four hours after treatment with RV-38AS, K562 cells show activation of the apoptotic pathway by the activation of both caspase- 8 and caspase- 9 . The fact that K562 cells undergo classical apoptosis after RV-38AS-induced cell cycle arrest is further demonstrated by the occurrence of nuclear condensation and fragmentation shown in both the light the electron micrographs by the fragmented DNA in DNA gel electrophoresis. Clearly, expression of Bcr-Abl AS RNA in K562 cells led to apoptosis induction after cell cycle arrest.

To further understand how the activation of PKR effectors regulate apoptosis induction, the underlying mechanism by which the apoptotic machinery integrates these signals were explored in the present study. Previous studies $(9,10)$ have already demonstrated that PKR-induced apoptosis involves mainly the FADD/caspase-8 pathway, although APAF/ caspase-9 activation is also observed during PKR-induced apoptosis (12). In light of these findings, we examined the possible involvement of caspase- 8 and caspase- 9 in the induction of apoptosis induced by the expression of the Bcr-Abl AS RNA. Results from our experiments showed elevated activation of both caspase- 8 and caspase- 9 during apoptosis induced by PKR activation, which is in good concordance with all the above findings. Meanwhile, in the present study, activation of caspase- 8 and caspase- 9 in K562 cells were found to increase with time kinetics similar to the Bcr-Abl AS RNA-induced PKR activation and cell apoptosis, suggesting a caspase- 8 and caspase-9 mediated apoptotic pathway in K562 cells. In addition, cells treated with the caspase- 8 inhibitor, Z-IETD-FMK, had a minor reduction of caspase-9 activity, indicating that caspase- 9 functions downstream of caspase- 8 in the PKR-induced apoptosis, which coincide with the findings of Iordanov et al (11) and Gil et al (12).

It has widely been accepted that apoptosis is an active gene-directed cellular suicide mechanism and many human genes, such as the p53 gene, c-Myc and the Bcl-2 gene family contribute to its regulation. Among these genes, Bcl-2 draws particular attention because it may be one of the key factors of the common final pathway involved in the regulation of cell apoptosis (26). BCL-2 can form heterodimers with BAX, a BCL-2-associated protein that antagonizes BCL-2 function and induces cell apoptosis (27). Meanwhile, PKR has previously been found to induce the expression of the pro-apoptotic protein BAX (10). In this study, we found that expression of Bcr-Abl AS RNA potently up-regulated BAX expression at both the mRNA and protein levels. No influences were found on the expression of BCL-2. The resulting net effect could thus lead to a lower ratio of BCL-2/BAX, which might be responsible for the RV-38AS-induced apoptosis in K562 cells.

Our study indicated that expression of Bcr-Abl AS RNA could selectively activate PKR in Bcr-Abl positive cells, which were further demonstrated to be sensitive to apoptosis induced by PKR activation. Furthermore, the activation of both caspase- 8 and caspase- 9 and a decrease in the BCL-2/BAX ratio, which are events downstream of apoptosis, were observed in K562 cells infected with the Bcr-Abl AS RNA-encoding retrovirus, which were further found to be antagonized by 2-AP.

Taken together, we demonstrate for the first time that the dsRNA-mediated cell killing strategy could selectively induce apoptosis of cultured K562 cells with ectopic expression of the Bcr-Abl AS RNA complementary to the unique e14a2 junction of Bcr-Abl through the activation of both caspase- 8 and 
caspase-9 pathways and the up-regulation of the pro-apoptotic factor, BAX. Therefore, the dsRNA-mediated cell killing strategy could be explored as a promising anti-leukemia agent or an adjuvant to the conventional therapeutic modalities in CML.

\section{Acknowledgements}

This study was supported by the research grant no. 30470744 from the National Science Foundation of China. We thank Professor Tong-Chuan He, the Director of the Molecular Oncology Laboratory of the University of Chicago Medical Center in America, for critical reading of the manuscript.

\section{References}

1. Sawyers CL: Opportunities and challenges in the development of kinase inhibitor therapy for cancer. Genes Dev 17: 2998-3010, 2003.

2. Ren R: Mechanisms of BCR-ABL in the pathogenesis of chronic myelogenous leukaemia. Nat Rev Cancer 5: 172-183, 2005.

3. Melo JV and Deininger MW: Biology of chronic myelogenous leukemia-signaling pathways of initiation and transformation. Hematol Oncol Clin North Am 18: 545-568, vii-viii, 2004.

4. Shah NP and Sawyers CL: Mechanisms of resistance to STI571 in Philadelphia chromosome-associated leukemias. Oncogene 22: 7389-7395, 2003.

5. Gorre ME, Mohammed M, Ellwood K, et al: Clinical resistance to STI-571 cancer therapy caused by BCR-ABL gene mutation or amplification. Science 293: 876-880, 2001.

6. Donato NJ, Wu JY, Stapley J, et al: Imatinib mesylate resistance through BCR-ABL independence in chronic myelogenous leukemia. Cancer Res 64: 672-677, 2004.

7. Garcia MA, Gil J, Ventoso I, et al: Impact of protein kinase PKR in cell biology: from antiviral to antiproliferative action. Microbiol Mol Biol Rev 70: 1032-1060, 2006.

8. Donze O, Deng J, Curran J, Sladek R, Picard D and Sonenberg N: The protein kinase PKR: a molecular clock that sequentially activates survival and death programs. EMBO J 23: 564-571, 2004.

9. Gil J and Esteban M: The interferon-induced protein kinase (PKR), triggers apoptosis through FADD-mediated activation of caspase 8 in a manner independent of Fas and TNF-alpha receptors. Oncogene 19: 3665-3674, 2000.

10. Balachandran S, Kim CN, Yeh WC, Mak TW, Bhalla K and Barber GN: Activation of the dsRNA-dependent protein kinase, PKR, induces apoptosis through FADD-mediated death signaling. EMBO J 17: 6888-6902, 1998.
11. Iordanov MS, Ryabinina OP, Schneider P and Magun BE: Two mechanisms of caspase 9 processing in double-stranded RNAand virus-triggered apoptosis. Apoptosis 10: 153-166, 2005.

12. Gil J, Garcia MA and Esteban M: caspase 9 activation by the dsRNA-dependent protein kinase, PKR: molecular mechanism and relevance. FEBS Lett 529: 249-255, 2002.

13. Shir A and Levitzki A: Inhibition of glioma growth by tumorspecific activation of double-stranded RNA-dependent protein kinase PKR. Nat Biotechnol 20: 895-900, 2002.

14. Shir A, Friedrich I and Levitzki A: Tumor specific activation of PKR as a non-toxic modality of cancer treatment. Semin Cancer Biol 13: 309-314, 2003.

15. Friedrich I, Ben-Bassat $\mathrm{H}$ and Levitzki A: Activation of dsRNA dependent protein kinase PKR in Karpas299 does not lead to cell death. Cancer Biol Ther 4: 734-739, 2005.

16. Futreal PA, Coin L, Marshall M, et al: A census of human cancer genes. Nat Rev Cancer 4: 177-183, 2004.

17. Manche L, Green SR, Schmedt C and Mathews MB: Interactions between double-stranded RNA regulators and the protein kinase DAI. Mol Cell Biol 12: 5238-5248, 1992.

18. Bevilacqua PC, George CX, Samuel CE and Cech TR: Binding of the protein kinase PKR to RNAs with secondary structure defects: role of the tandem A-G mismatch and noncontiguous helixes. Biochemistry 37: 6303-6316, 1998.

19. He Y and Huang L: Growth inhibition of human papillomavirus 16 DNA-positive mouse tumor by antisense RNA transcribed from U6 promoter. Cancer Res 57: 3993-3999, 1997.

20. Herrmann M, Lorenz HM, Voll R, Grunke M, Woith W and Kalden JR: A rapid and simple method for the isolation of apoptotic DNA fragments. Nucleic Acids Res 22: 5506-5507, 1994.

21. Srivastava SP, Kumar KU and Kaufman RJ: Phosphorylation of eukaryotic translation initiation factor 2 mediates apoptosis in response to activation of the double-stranded RNA-dependent protein kinase. J Biol Chem 273: 2416-2423, 1998.

22. Gil J, Alcami J and Esteban M: Induction of apoptosis by double-stranded-RNA-dependent protein kinase (PKR) involves the alpha subunit of eukaryotic translation initiation factor 2 and NF-kappaB. Mol Cell Biol 19: 4653-4663, 1999.

23. Venkataraman S, Dirks RM, Ueda CT and Pierce NA: Selective cell death mediated by small conditional RNAs. Proc Natl Acad Sci USA 107: 16777-16782, 2010.

24. Zamanian-Daryoush M, Der SD and Williams BR: Cell cycle regulation of the double stranded RNA activated protein kinase, PKR. Oncogene 18: 315-326, 1999.

25. Petryshyn RA, Li J and Judware R: Activation of the dsRNAdependent kinase. Prog Mol Subcell Biol 14: 1-14, 1994.

26. Hockenbery DM, Oltvai ZN, Yin XM, Milliman CL and Korsmeyer SJ: Bcl-2 functions in an antioxidant pathway to prevent apoptosis. Cell 75: 241-251, 1993.

27. Oltvai ZN, Milliman CL and Korsmeyer SJ: Bcl-2 heterodimerizes in vivo with a conserved homolog, Bax, that accelerates programmed cell death. Cell 74: 609-619, 1993. 